\title{
A Sequence Analysis of Nonverbal Behaviour and Deception
}

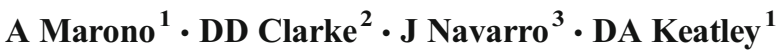

Published online: 7 June 2017

(C) The Author(s) 2017. This article is an open access publication

\begin{abstract}
The ability to correctly interpret nonverbal communication (NVC) is an important ability in everyday interactions, which may use NVC techniques to identify the concealment of information. In the present study, a novel approach was used to understand NVC. Behaviour sequence analysis identified specific sequences of behaviours that indicate psychological distress caused by deception. The study involved the analysis of 55 videos of real criminals and high-power individuals that were filmed fabricating statements, which were later exposed as being untruthful at the time of being filmed. In addition, 53 clips of criminals making truthful statements were also analysed as a contrast group. Results indicated clear differences between honest and deceptive responses, such as furrowing of eyebrows in the deceptive sequences occurring more often than honest statements. In addition, sequences of behaviours were shown in the present data set, which could indicate a new method for analysing NVC and detecting psychological distress caused by deception. The possible implications and applications for police and forensic investigation are also outlined.
\end{abstract}

Electronic supplementary material The online version of this article (doi:10.1007/s11896-017-9238-9) contains supplementary material, which is available to authorized users.

DA Keatley

DKeatley@lincoln.ac.uk

J Navarro

http://www.jnforensics.com

1 Researchers in Behaviour Sequence Analysis, Psychology Department, University of Lincoln, Lincoln LN67TS, UK

2 Researchers in Behaviour Sequence Analysis, Psychology Department, University of Nottingham, Nottingham NG72RD, UK

3 JN Forensics, LCC, Tampa, FL, USA
Keywords Nonverbal communication · Behaviour sequence analysis $\cdot$ Credibility assessment $\cdot$ Deception $\cdot$ Suspect behaviour

\section{Introduction}

Understanding nonverbal communication (NVC) is important in a range of situations and settings, from daily relationships to interrogations (Krauss et al. 1996). Research has shown that NVC includes both reflexive and nonreflexive movements of the body, which communicate an emotional message to others (Tamietto and De Gelder 2010). Correct interpretation of individuals' emotional states and intentions is obviously important for effective communication; however, a particularly important area for NVC is in police and legal investigations. During a police interview or interrogation, investigators may implicitly or explicitly use NVC reading techniques to identify the concealment of emotions, which may indicate an intent to deceive or a line of questioning worth pursuing (Hartwig and Bond 2011; Mann et al. 2004). Behavioural cues of mental effort, memories and emotions are used by security personnel to identify behavioural 'hot spots' that indicate that a topic is worth considering and investigating further (Frank et al. 2008). Typically, research has focused on behaviours related to particular emotions or concealment of information (Ekman and O'Sullivan 1991). Sporer and Schwandt (2007) outline several reliable indicators of deception, such as nodding, movement of feet and legs and hand movements; however, they showed no systematic relationship between avoidance of eye contact and deception. However, researchers and professional practitioners in the field have suggested that clusters of behaviours are better indicators of changes in emotion and possible psychological discomfort, which may arise from attempting to deceive someone (DePaulo et al. 2003; 
Navarro and Karlins 2008, Navarro 2011; Vrij et al. 2004). The present research continues a more recent trend in NVC by investigating the possibility that it is not only discrete behaviours or clusters of movements that can be used to indicate emotion or distress but also the sequence that these movements occur in (Burgoon et al. 2014; Burgoon et al. 2015). Previous research that has focused on sequences of behaviour has used a software programme that detects patterns in timeordered data. These T-patterns (see Casarrubea et al. 2015) are a multivariate approach to identifying temporal structure in behaviour. Burgoon et al. (2014), therefore, used a very similar statistical approach to the one taken in the current study; however, their series of studies focused on mock theft, cheating game behaviours and group discussions and deception. The current study uses a similar statistical approach, but with real-world deception.

Understanding and interpreting NVC typically start by identifying discrete, single behaviours, referred to as tells (Collett 2003; Ekman et al. 1991; Navarro and Karlins 2008). Hartwig and Bond (2011) outlined the leakage hypothesis as a means by which individuals leak informative tells while trying to conceal the truth. For instance, poker players who have become successful at reading NVC have the ability to identify particular behaviours of their opponents. This line of research, however, has limitations in terms of individual differences in the ability to mask emotion and deception (Blanck et al. 1981).

In contrast to looking at single behaviours or micro reactions, researchers have suggested that 'clusters' of behaviours are better indicators of changes in emotion and psychological distress, which may reflect attempts to deceive (DePaulo et al. 2003; Hartwig and Bond 2011; Navarro and Karlins 2008; Vrij et al. 2004). Therefore, simply recognising one single behaviour as an indication of emotions or lying is not completely reliable, though Hartwig and Bond (2011) highlight that while multiple cues may be better than single cues, individual behaviours still contribute a lot to the detection of deception. The cluster approach, which is based on more applied settings, outlines that NVC involves the changes of behaviours across the entire body (i.e., movement of feet, hands, face, etc.). This research has gained support from applied fields of forensic and investigative psychology and police work (Furnham and Taylor 2011). While the cluster approach has proven effective, there may be an additional step in terms of understanding the sequence of body movements. For instance, it may be possible that body movements occur in sequence (i.e., the feet change movement first, followed by the face). Research has shown that if an individual attempts to mask one behaviour, they may leak behaviours on other parts of their body (Ekman and Friesen 1969). Looking at patterns of behaviours in deceptive and truthful interactions has been previously researched by
Burgoon et al. $(2014,2015)$. In their seminal research, patterns of nonverbal behaviour, across time, were analysed, showing clear differences between deceivers and truthful participants. Burgoon and colleagues' research provides a clear foundation to begin future development in investigating patterns and sequences of behaviours, rather than individual behaviours or clusters. It is to this direction the present research builds on, by using real-world deceptive statements, rather than mock crime experiments.

\section{Behaviour Sequence Analysis}

Behaviour sequence analysis (BSA), also referred to as lag sequence analysis (LSA), is a useful method for understanding the dynamic relationship between progressions of behaviours and social interactions occurring over time (Beune et al. 2010; Keatley et al. 2016; Taylor et al. 2008). Sequence analysis typically involves three key stages (Clarke and Crossland 1985). First, unitisation involves taking a person's whole response to a question and cutting it into discrete behaviours or events. Second, classification involves placing the behaviours or events into distinct categories that are functionally similar. Finally, analysis involves the statistical measurement of whether transitions between behaviours are occurring significantly above the level of chance. For example, if we imagine a simple chain of behaviours, question asked (category $a$ ), respondent shakes head (category $b$ ), respondent taps finger (category $c$ ) and respondent looks up and right (category $d$ ), first-order or lag-one behaviour sequence analysis tests whether 'a-b', 'a-c', b-c' and 'c-d' pairs, for instance, are more or less likely to occur than by chance alone. Therefore, sequence analysis begins by first finding stimuli (e.g., video clips of individuals caught lying or telling the truth) that can be analysed. A coding scheme is then developed so that each individual behaviour shown in the stimuli can be categorised (e.g., 'head-nod', 'eyes-looks down'). When an exhaustive and mutually exclusive list is developed, all stimuli are then coded. The sequence analysis then measures transitions between pairs of behaviours, to see if pairs occur above chance.

BSA is underpinned by Markov models, which involve studying transitions between behaviour pairs (Ivanouw 2007). The first event in a pairing is the antecedent (e.g., nods head); the second behaviour in a pair is the sequitur (e.g., taps finger). In the simplest form of BSA, the analysis determines whether the antecedent causes the sequitur to be more likely to occur, than expected by chance. Sequence analysis has been used in a variety of social interactions and behaviours, such as marital conflict (Gottman 1979), violent episodes between people (Beale et al. 1998; Turner and Clarke 2009) and rape cases, in relation to physical interactions (Fossi et al. 2005) as well as verbal interactions and strategies (Lawrence et al. 2010) between attacker and victim. 


\section{Present Study}

The main aim of the current research is to provide a new method, behaviour sequence analysis, for understanding and researching NVC. Previous research has shown that the accuracy rates of trained professionals are modest, in terms of detecting deception through NVC techniques (Burgoon et al. 2014; Burgoon et al. 2015; Frank and Ekman 1997; Vauch et al. 2016). However, many NVC studies lack ecological validity (Richardson et al. 2000; Scriba et al. 1999). Although participants in lab-based experiments may be motivated towards an outcome, they are not at risk of major negative consequences for being exposed, as a criminal would be in a real investigation. Indeed, the absence of such high stakes might not produce a sufficient amount of anxiety or motivation for the person in question to display valid facial cues or NVC (Miller and Stiff 1993). Therefore, the current study used real-world recorded videos of people, rather than in a laboratory. The sample chosen were criminals, and/or people of high power, who were later unequivocally exposed as being guilty at the time of making the statements of innocence that were recorded and analysed. Owing to the nature of the current study, no formal hypotheses were made. However, it is likely that previous research into NVC will be supported as elements of larger sequences. There are also likely to be differences in the chains of behaviours exhibited between deceptive and truthful statements. Based on the research by Hartwig and Bond (2011) and Sporer and Schmidt (2007), behaviours related to head movements and hand movements are likely to be different between honest and dishonest statements, and clusters of behaviours are likely to occur, but with certain individual behaviours occurring more frequently in honest and dishonest statements.

\section{Methods}

\section{Sample}

Video-recorded interview clips of politicians, criminals and people of high interest were obtained through various media sources. Content of these clips were real-life examples of deception or honesty. Each clip contained statements made by participants in response to a question, which were later proven to be a lie or the truth. Clips came from popular online video websites and included scenes from press conferences through to police interrogations. A sample of videos of 19 individuals ( 15 male, 4 female) between the ages of 19-48 was collected via online websites and archive footage documents, for both groups. Multiple clips were used for the same individuals, in some cases. For the truthful response group, 12 criminals were analysed (11 males, 1 female). ${ }^{1}$ For the deception group, a total of 51 clips were used. Length of clips ranged from 4 to $58 \mathrm{~s}(M=16.05, \mathrm{SD}=11.64)$. For the truthful group, a total of 49 clips were used. Length of clip ranged from 6 to $57 \mathrm{~s}(M=18.6, \mathrm{SD}=10.2)$. Clips were obtained through cross-referencing well-known cases of individuals caught lying with existing recordings of incidents in which they are clearly shown on tape lying. For both groups, lying about committing murder was the most frequent video clip used ( $n=10$ in dishonest group, $n=6$ in honest group).

Inclusion criteria for each group were that the person was unequivocally exposed as lying or telling the truth by later investigation and evidence. Everyone in the study had no indication of any medical condition that would affect their body movements. The study was approved by the University of [omitted] Research Ethics Committee.

\section{Coding Procedure}

Behaviours were recorded in relation to a question and answer pattern. A question was asked; then once the participant began to answer with a response, behaviours were recorded until the end of the response. When a new question was asked, a new sequence of behaviours was analysed. Initially, a list of possible behaviours was developed from existing literature (Burgoon et al. 2014, 2015). This provided a baseline of behaviours that might occur when viewing the clips. ${ }^{2}$ If any additional behaviours became apparent when viewing the clips, these behaviours were added to the list. Clips were slowed down and viewed frame by frame, in order to get a more accurate analysis of the sequence of NVC. The coding categories were mutually exclusive and exhaustive, which is a prerequisite of sequence analysis (Bakeman and Quera 2011). Each clip was analysed by two separate researchers involved in the study to ensure inter-rater reliability. Finally, the coding scheme was given to an expert in the field to assess and approve. Given the nature of the coding and behaviour sequence analysis, future research can be directly added to the current data to build larger data sets for analyses.

\section{Statistical Analysis}

After videos were coded into chains of discrete categories, data were input into the statistical software R (R Core Team

\footnotetext{
${ }^{1}$ For descriptions of individuals used in the analysis, topic of statement, and length of clip, see supplementary material S1. It should be noted that different individuals were used for honest and deception groups, owing to availability of materials. However, both groups contained criminals - to allow better comparison. Sampling was therefore purposeful, focusing on only those videos that were of high quality and of individuals who were dishonest or honest.

${ }^{2}$ As video clips only showed clear movements from the chest-upwards for the majority of people, lower limbs were not put into the sequence analysis. See Supplementary material S2 for coding list.
} 
Table 1 Frequencies of behaviours - deception statements

\begin{tabular}{lc}
\hline Behaviour & Frequency \\
\hline Head-shake head & 66 \\
Eyes-looks down & 58 \\
Head-nod head & 53 \\
Face-furrow eyebrows (pull brows down) & 44 \\
Face-raised eyebrows & 39 \\
Mouth-pressing lips together & 22 \\
Head-tilts head sideways & 21 \\
Eyes-irregular blinking & 20 \\
Eyes-looks to the side & 17 \\
Head-tilt head forward; eyes-looks up & 14 \\
Mouth-full mouth smile & 13 \\
Head-tilts head down & 12 \\
Eyes-avert eyes & 10 \\
Body-half shrug; mouth-licking lips; mouth-harsh swallow & 9 \\
Face-tightening jaw; hands-clenching fists; hands-slams hands down; mouth-pouting (push lips & 8 \\
$\quad$ forward and together); head-circle head &
\end{tabular}

Behaviours are coded by body location (e.g., face, eyes, body) followed by what the behaviour/movement was that occurred (e.g., twitch, look away, open mouth). Behaviours with the same frequencies are grouped together
2013) and analysed using a behaviour sequence analysis programme that the researchers wrote. The programme calculated frequencies of individual behaviours, transitional frequencies, chi-squared $\left(\chi^{2}\right)$ statistics and standardised residuals.

\section{Results}

\section{Deception Sequences}

Analyses were conducted on 55 video sequences of a particular question-answer episode in which participants were exposed to be lying. Frequencies of behaviours that participants showed were first calculated (see Table 1). The most frequently occurring behaviours were participants shaking their head $(n=66)$, looking down $(n=58)$, nodding their head $(n=53)$ and furrowing their eyebrows (pulling their eyebrows down) $(n=44)$. These results support previous findings on deception related to NVC behaviours (Mann et al. 2002) (Table 2).
The next, main stage of sequence analysis is to calculate transition frequencies between antecedents and sequiturs. A state transition diagram ${ }^{3}$ was made on the data (see Fig. 1). The first thing to note with the state transition diagram is that a first-order, also known as lag one, sequence analysis, was conducted. Therefore, only links between behaviour pairs are analysed. ${ }^{4}$ These pairs then form longer chains. The correct way to read the diagram is moving from one behaviour in single steps to a following behaviour, linked via an arrow. Whereas the diagram shows a full map from start to end, which is not to say any/all participants followed the same routes entirely, numbers beside the arrows indicate likelihood and frequency of transitions. Second, all transitions in the diagram are statistically significant $(p<0.05)$. Klonek and

\footnotetext{
3 Transition matrices are typically extremely large and are therefore available as Supplementary material S3.

${ }^{4}$ It would be possible to conduct 'higher order' sequence analysis (e.g., $\mathrm{AB} \rightarrow \mathrm{C}, \mathrm{BC} \rightarrow \mathrm{D}$ chains); however, this typically leads to over-fitting of data to particular stimuli, and typically does not offer clearer analyses.
} 
Table 2 Frequencies of behaviours - truthful statements

\begin{tabular}{lc}
\hline Behaviour & Frequency \\
\hline Eyes-looks up & 39 \\
Head-nod head & 35 \\
Face-raised eyebrows & 27 \\
Eyes-looks to the side & 26 \\
Face-keep a 'frozen' face & 20 \\
Head-tilts head up & 19 \\
Face-furrow eyebrows (pull brows down) & 15 \\
Eyes-looks down & 14 \\
Mouth-harsh swallow; head-tilts head sideways & 13 \\
Eyes-widen eyes; mouth-pouting (push lips forward and together); mouth-pressing lips together & 12 \\
Body-slouching; head-tilt head forward & 11 \\
Hands-palms outwards & 10 \\
Face-flair nostrils & 9 \\
Face-tightening jaw & 8 \\
Mouth-licking lips; body-creating physical barrier & 6 \\
Face-self-touch (eyes); eyes-irregular blinking; body-straightening up; hands-reach hand out; & 5 \\
body-pointing (away); mouth-opens mouth & 5 \\
Eyes-avert eyes; mouth-full mouth smile; head-tilts head down & 4 \\
Fody-fidget; body-tilts forwards; body-slopping shoulders & 4 \\
Face-self-touch (mouth); body-shrugging; hands-clenching fists; body-crossing arms; & 3 \\
$\quad$ body-tapping foot; body-self-touch (other/body); head-circle head; body-puffed chest; & 2 \\
eyes-look towards exit & 2 \\
\hline
\end{tabular}

Behaviours are coded by body location (e.g., face, eyes, body) followed by what the behaviour/movement was that occurred (e.g., twitch, look away, open mouth). Behaviours with the same frequencies are grouped together colleagues suggest a cut-off criteria of transitional frequencies below 5 being omitted from analyses for large data sets (Klonek et al. 2015); however, given the smaller data set of the current sample, a frequency cut-off of only showing transitions above frequency of 3 was used, to reduce complexity and only include meaningfully high transitions.

The diagram should be read one step at a time, between behaviours. For instance, after being asked a question, furrowing the eyebrows has a standardised residual (SR) of 1.2, and six individuals made that response, in the current data set. The $n$ values in the diagram refer to the representation of transitions across the video clips, rather than individual people. After eyebrows have been furrowed, several different behaviours followed, for different individuals. For instance, after furrowing the eyebrows, head shaking is two times more likely to occur than chance, and nine individuals showed that link. These links illustrate the pairings of behaviours, rather than long (multiple behaviour) sequences. Of the original six people who showed furrowing eyebrows, several may have shown other behaviours next, not directly moving on to head shaking. Therefore, a first-order, or lag-one state transition, diagram shows only links between behaviour pairs, not longer chains.
Figure 1 shows that overall, the first significant behaviour displayed after a question has been asked, is the furrowing of eyebrow ( $\mathrm{SR}=1.2, n=6$ ). This behaviour leads to several following behaviours: head tilt sideways ( $\mathrm{SR}=1.2, n=3$ ), slam hands down ( $\mathrm{SR}=3.2, n=3)$, tilt head forward ( $\mathrm{SR}=2.0, n=3)$, shake head $(\mathrm{SR}=2.0, n=9)$ and eyes look down ( $\mathrm{SR}=1.4, n=7)$. Head shaking was the most frequently occurring behaviour and had a direct link to end of the sequence (i.e., end of answering) ( $\mathrm{SR}=3.6, n=14$ ). This link was the most frequently occurring link in the diagram. This suggests that the final gesture individuals make before finishing a lying sequence was to shake their head. The behaviour 'eyes-looking down' was the next most frequently occurring behaviour, but was not directly linked to the end of the answer sequence. Instead, several individuals ended their answer sequence by nodding their head $(\mathrm{SR}=1.2$, $n=7$ ) or pressing lips together ( $\mathrm{SR}=3.1, n=6$ ), with slightly fewer tightening their jaw (SR $=4.1, n=4)$ or simply tilting head forward (perhaps in a half nod gesture) $(\mathrm{SR}=1.2, n=7)$.

\section{Truthful Sequences}

Analyses were also conducted on truthful responses. Frequencies of behaviours exhibited by honest respondents were 


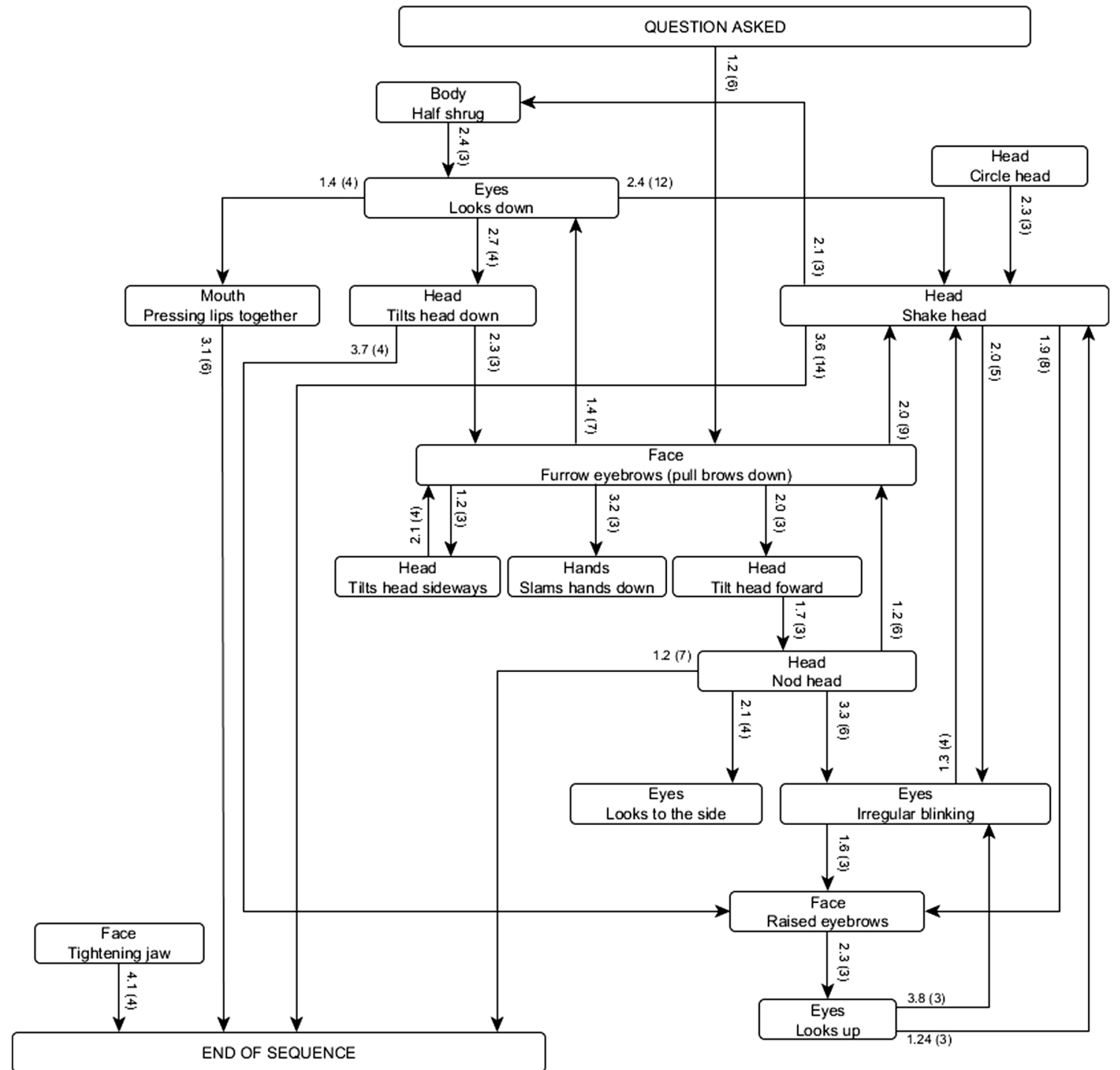

Fig. 1 State transition diagram of behaviours exhibited during question response for deception sequences. All transitions shown are significant. Standardised residuals are given above or beside arrows; frequencies are

calculated first (see Table 1). The most frequently occurring behaviours were eyes-looks up $(n=39)$ and head-nods $(n=35)$.

The next main stage of sequence analysis, to allow a contrast between deceptive and honest movements, was to conduct a sequence analysis on the honest group. The first notable difference between the two sequences is that the honest sequence state transition diagram has fewer behaviour exhibited. Within the sequences, there is no clear path between start and end of response; however, head tiling forward $(\mathrm{SR}=4.1$, $n=6)$ and head tilting sideways ( $\mathrm{SR}=2.0, n=4)$ were the first significant behaviours exhibited by honest respondents, which is in contrast to dishonest responses, wherein furrowing of the eyebrows preceded both behaviours (Fig. 2). shown in brackets. Position of boxes does not indicate temporal orderarrows indicate sequence of behaviours

\section{Discussion}

The aim of the current research was to show the benefits of a behaviour sequence analysis approach to understanding NVC in relation to deception. The first part of the BSA, which shows frequencies of individual behaviours, indicates support for previous findings in the literature (Mann et al. 2002). The frequency analyses also indicate that deceptive individuals repeatedly demonstrated one behaviour particular to them, aka their 'tell', which they tended to repeat regularly when making a lying statement; this is why head shake, for instance, occurs so frequently. In contrast, honest responses typically exhibited fewer behaviours overall, which supports previous findings in the 
Fig. 2 State transition diagram of behaviours exhibited during question response for truthful sequences. All transitions shown are significant. Standardised residuals are given above or beside arrows; frequencies are shown in brackets. Position of boxes does not indicate temporal order-arrows indicate sequence of behaviours

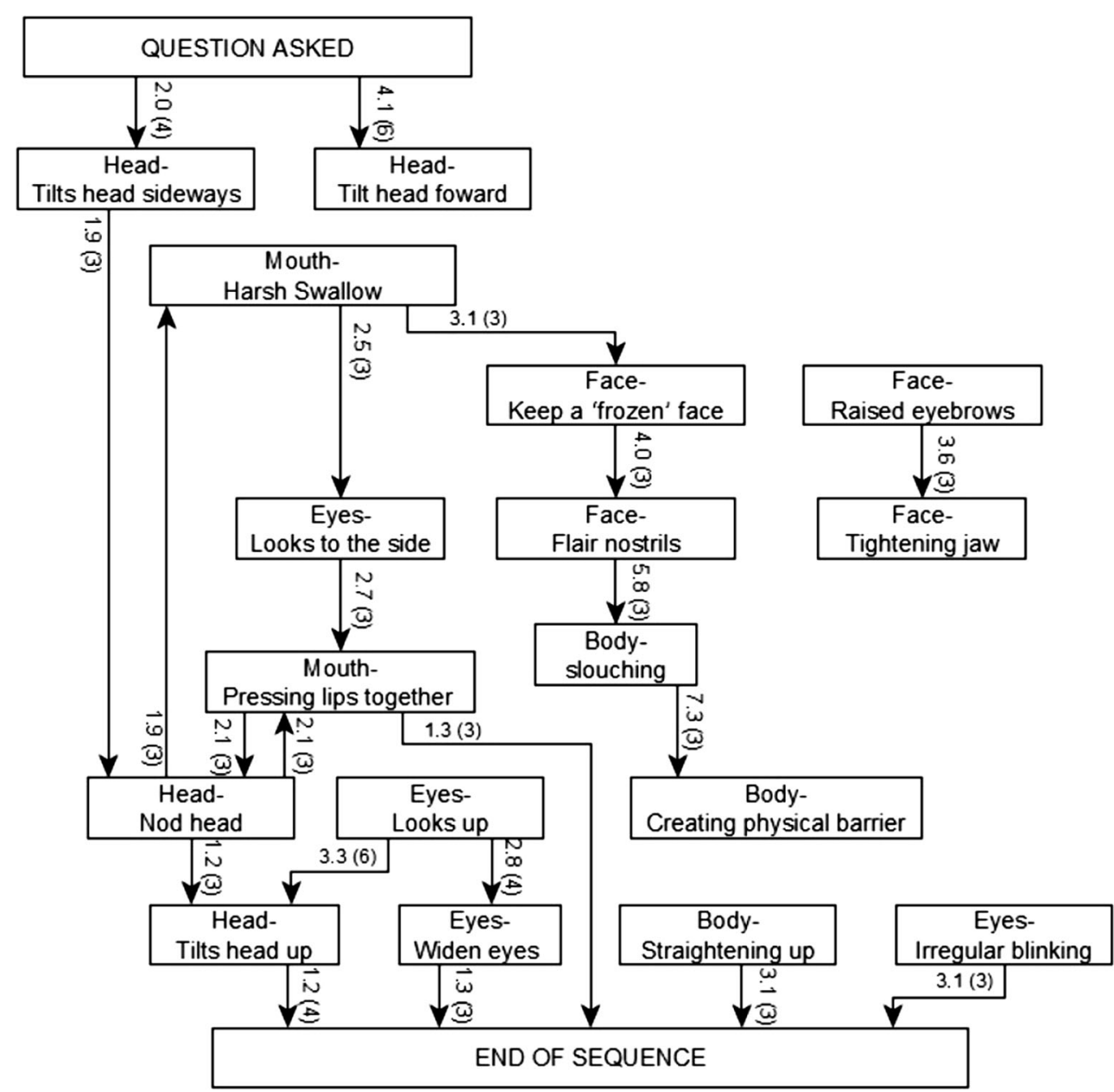

literature (Watson et al. 2016). In this way, the frequencies in the current research are analogous to previous research that has focused on individual tells. However, as Hartwig and Bond (2011) suggest, multiple cues may be better indicators of deception and honesty. Therefore, the next stage of the research was to investigate the sequence of behaviour pairs.

The state transition diagram for the deception sequences shows that the first behaviour following being asked a question was furrowing of the eyebrows. This may be an indicator of individuals needing to stop and think about their answer (Schmidt and Cohn 2001). This is also in contrast to the honest sequence diagram, in which furrowing of the eyebrows did not occur at all. In the deception sequences, several behaviours followed, which indicate further consideration and possible reflection. For instance, the head tilt sideways linked directly back to furrowing of the eyebrows and may be the case that these behaviours show an internal conflict requiring attention and consideration (Schmidt and Cohn 2001). This shows that head tilting sideways is not, in itself, an indicator of deception.
In contrast to people shaking their heads, it may be that some individuals are aware of this tell and deliberately nodded instead, to mask their knowledge. This finding indicates that nodding or shaking of the head alone are not conclusive indicators of deceit, which supports previous literature in the area of NVC (Mann et al. 2002). The role of eye movements was not clearly supported in the current findings, which supports the research of Sporer and Schwandt (2007). It should be noted that the current sample involved people who may be aware and deliberately masking body movements. Indeed, a strength of the cluster approach and sequence analysis approach to NVC is that is capitalises on tracking and highlighting multiple behaviours individuals might accidently make or leak when concentrating too much on controlling a particular body part.

There are several limitations and areas of future research stemming from the present study. First, the current analysis used a behaviour sequence approach to understand the progression of behaviours, which did not account for time, per se. 
Future research could focus on time-interval sequences, which might clarify the changes between micro tells (occurring very early in the sequence) compared to deliberative body movements (occurring after a few seconds have elapsed). A further limitation was the use of behaviours above chest height (i.e., mainly the face). Also, baseline measures were not an option in the current recordings, which were clips of only the lying answer. A further limitation was that multiple clips from the same person have been used in the current data set, which could lead to over-representation of particular cues. Future research should build on the current findings with different individuals, to reduce the potential impact of this issue. Future research should also include more women and men so that gender differences can be analysed. Future research could investigate a 'pre-question' sequence of behaviours and analyse whether there are significant changes in behaviours, which could indicate changes in emotion or deceit. A major strength of the current research, beyond the new method introduced, was the use of real-world data. The majority of NVC and deceit research is conducted in laboratory settings; the current method allows for real-world behaviours to be analysed. This also provides an opportunity for future research to compare sequences of actual behaviours caught on film compared to lab study behaviours. The outcome of this comparison might show that lab studies show very different sequences of behaviours, in contrast to real-world cases. Finally, future research could focus on verbal alongside nonverbal communication. Research using BSA has previously focused on verbal strategies (Lawrence et al. 2010), however, not in relation to deceitfulness of the statements. Therefore, a much needed progression in the literature is to investigate the role of verbal and nonverbal lies.

\section{Conclusions}

The current research will not only help to understand what behaviours are produced when a deceptive statement is constructed, but it may also determine the sequences of production and deduce potential critical behaviours within larger sequences of nonverbal chains. The sequence analysis approach allows us to see very clearly, changes in behaviours and NVC, which are an indication of changes within the individual as a result of the question that has been asked. This approach is a novel way of statistically analysing changes in behaviour and indicating what this may mean, for example, changes in emotion or reasons for emotional changes. Investigating temporal changes in NVC could be a useful investigation tool for interviewers or interrogators to use when analysing responses. Sequences of behaviours could be used to identify changes in behaviour as a reaction to questions and therefore indicate where to focus future further questioning, to investigate the cause of the behaviours.

\section{Compliance with Ethical Standards}

Funding (If any): No funding was received for the current research.

Conflict of Interest The authors declare that they have no conflict of interest.

Ethical Approval Ethics was approved by the School of Psychology Research Ethics Committee at the University of [removed for anonymous review].

Informed Consent No participants were used in the current study, as all materials were freely accessibly from online media sites. If ownership of videos was in dispute, consent for the use would have been given; however, as all materials were available on multiple online free sites, it was deemed appropriate to use for analysis by the Ethics Committee.

Open Access This article is distributed under the terms of the Creative Commons Attribution 4.0 International License (http:// creativecommons.org/licenses/by/4.0/), which permits unrestricted use, distribution, and reproduction in any medium, provided you give appropriate credit to the original author(s) and the source, provide a link to the Creative Commons license, and indicate if changes were made.

\section{References}

Bakeman R, Quera V (2011). Sequential analysis and observational methods for the behavioral sciences. Cambridge University Press, Cambridge

Beale D, Cox T, Clarke DD, Lawrence C, Leather P (1998) Temporal architecture of violent incidents. J Occup Health Psychol 3:65-82

Beune K, Giebels E, Taylor PJ (2010) Patterns of interaction in police interviews: the role of cultural dependency. Crim Justice Behav 37: 904-925

Blanck PD, Rosenthal R, Snodgrass SE, DePaulo BM, Zuckerman M (1981) Sex differences in eavesdropping on nonverbal cues: developmental changes. J Pers Soc Psychol 41:391-396

Burgoon JK, Schuetzler R, Wilson D (2015) Kinesic patterning in deceptive and truthful interactions. J Nonverbal Behav 39:1-24

Burgoon JK, Proudfoot JG, Schuetzler R, Wilson D (2014) Patterns of nonverbal behavior associated with truth and deception: illustrations from three experiments. J Nonverbal Behav 38:325-354

Casarrubea M, Jonsson GK, Faulisi F, Sorbera F, Di Giovanni G, Benigno A, Crescimanno G, Magnusson MS (2015) T-pattern analysis for the study of temporal structure of animal and human behavior: a comprehensive review. J Neurosci Methods 239:34-46

Clarke, D. D., \& Crossland, J. (1985). Action systems: an introduction to the analysis of complex behaviour, London: Methuen.

Collett P (2003) The book of tells. Bantam, New York

DePaulo BM, Lindsay JJ, Malone BE, Muhlenbruck L, Charlton K, Cooper H (2003) Cues to deception. Psychol Bull 129:74-118

Ekman P, Friesen WV (1969) Nonverbal leakage and clues to deception. Psychiatry 32:88-106

Ekman P, O’Sullivan M (1991) Who can catch a liar? Am Psychol 46:913

Ekman P, O'Sullivan M, Friesen WV, Scherer KR (1991) Face, voice, and body in detecting deception. J Nonverbal Behav 15:125-135

Fossi JJ, Clarke DD, Lawrence C (2005) Bedroom rape sequences of sexual behavior in stranger assaults. Journal of interpersonal violence 20:1444-1466

Frank MG, Ekman P (1997) The ability to detect deceit generalizes across different types of high-stake lies. J Pers Soc Psychol 72:1429 
Frank MG, Menasco MA, O'Sullivan M (2008) Human behavior and deception detection. In: Voeller JG (ed) Handbook of science and technology for homeland security, vol 5. John Wiley \& Sons, Inc., Hoboken

Furnham A, Taylor J (2011) Bad apples: identify, prevent \& manage negative behavior at work. Basingstoke, Palgrave Macmillan

Gottman JM (1979) Marital interaction: experimental investigations. Academic Press, New York

Hartwig M, Bond CF Jr (2011) Why do lie-catchers fail? A lens model meta-analysis of human lie judgements. Psychol Bull 137:643-659

Ivanouw J (2007) Sequence analysis as a method for psychological research. Nordic Psychology 59:251-267

Keatley, D. A., Barsky, A. D., \& Clarke, D. D. (2016). Drink driving in the UK: a sequence analysis approach. In: British Society for the Psychology of Individual Differences, 8 April 2016, Nottingham Trent University.

Klonek FE, Quera V, Kauffeld S (2015) Coding interactions in motivational interviewing with computer-software. Comput hum Behav 44(C):284-292

Krauss RM, Chen Y, Chawla P (1996) Nonverbal behavior and nonverbal communication: what do conversational hand gestures tell us? Adv Exp Soc Psychol 28:389-450

Lawrence C, Fossi J, Clarke D (2010) A sequential examination of offenders' verbal strategies during stranger rapes: the influence of location. Psychol Crime law 16:381-400

Mann S, Vrij A, Bull R (2002) Suspects, lies, and videotape: an analysis of authentic high-stake liars. Law hum Behav 26:365-376

Mann S, Vrij A, Bull R (2004) Detecting true lies: police officers' ability to detect suspects' lies. J Appl Psychol 89:137 Chicago

Miller GR, Stiff JB (1993) Deceptive communication, vol 14. Sage Publications, Inc., Newbury Park

Navarro J (2011) Clues to deceit: a practical list. A \& A Printing, Tampa

Navarro J, Karlins M (2008) What every BODY is saying, vol 27. Harper Collins, New York, p 90
R Core Team. (2013). R: a language and environment for statistical computing. R Foundation for Statistical Computing, Vienne, Austria. ISBN 3-900051-07-0, URL http://www.R-project.org/.

Richardson CK, Bowers D, Bauer RM, Heilman KM, Leonard CM (2000) Digitizing the moving face during dynamic displays of emotion. Neuropsychologia 38:1028-1039

Schmidt KL, Cohn JF (2001) Human facial expressions as adaptations: evolutionary questions in facial expression research. Am J Phys Anthropol 116:3-24

Scriba H, Stoeckli SJ, Veraguth D, Pollak A, Fisch U (1999) Objective evaluation of normal facial function. Annals of Otology, Rhinology \& Laryngology 108:641-644

Sporer SL, Schwandt B (2007) Moderators on non-verbal indicators of deception: a meta-analytic synthesis. Psychol Public Policy law 13: $1-34$

Tamietto M, De Gelder B (2010) Neural bases of the non-conscious perception of emotional signals. Nat rev Neurosci 11:697-709

Taylor PJ, Jacques K, Giebels E, Levine M, Best R, Winter J, Rossi G (2008) Analysing forensic processes: taking time into account. Issues in Forensic Psychology 8:45-57.57

Turner K, Clarke DD (2009) Aggression in intellectual disability - a new approach. Ment Health rev J 14:28-36

Vauch V, Sporer SL, Michael SW, Meissner CA (2016) Does training improve the detection of deception? A meta-analysis. Commun res 43:283-343

Vrij A, Akehurst L, Soukara S, Bull R (2004) Detecting deceit via analyses of verbal and nonverbal behavior in children and adults. Hum Commun res 30:8-41

Watson, S. J., Conchie, S. M., Taylor, P. J., \& Poppe, R. W. (2016). Utilising motion capture technology to identify trusted testimony in military encounters. Poster presented at the BPS $5^{\text {th }}$ Military Conference, Basingstoke, UK. 